\title{
A 3D Model Based Action Recorder Using Computer Vision
}

\author{
Yong $\mathrm{Li}^{1}$, Yuan $\mathrm{Rao}^{2}$, Youxiang Duan ${ }^{1}$, and Weishan Zhang ${ }^{1}$ \\ ${ }^{1}$ College of Computer \& Communication Engineering, China University of Petroleum \\ No. 66 Changjiang West Road, Huangdao District, Qingdao, China, 266580 \\ ${ }^{2}$ School of Software Engineering, Xi' an Jiaotong University, Xi' an, China, 710049 \\ \{evaleao, yxduan, zhangws\}@upc.edu.cn, raoyuan@mail.xjtu.edu.cn
}

\begin{abstract}
In healthcare systems, video surveillance, interactive games and many other applications, human action recognition and recording are very important. This paper presents a comprehensive 3D model based action recording system using computer visions. First, a computer captures human motion videos with a network camera and conducts further detection and tracking of the video resources, then a 3D model is created based on the recorded data results. The action recording system includes background capturing, characters capturing, action tracking, 3D modeling and OGRE controlling. OpenCV is used for background and characters capturing where a background image difference algorithm is used to analyze a moving target and extract different elements. For the action tracking, the Camshift (Continuously Adaptive Mean shift Algorithm) tracking algorithm is used to realize continuous tracking and recognition of moving objects and ensure good performance of the action recorder. In our implementation, 3Dmax is used to build a 3D model and skeletal animations, where Ogremax is used to export models, and then to import the skeletal animations into a testing environment. The evaluations show that our motion recognition and recording system has good performance in one aspect, and can obtain accurate result on the other aspect.
\end{abstract}

Keywords: Target detection and tracking, Background difference, Action recognition.

\section{Introduction}

Human computer interaction is very active due to computing devices are increasing more and more popular in everyday life. Human movements are usually captured using various kinds of sensors, and then the movement information is recorded to reproduce virtual actions. These sensors include mechanical, acoustic, electromagnetic and optical ones. For example, the SixthSense interactive technology based on OpenCV is a wearable gestural interface device invented by Pranav Mistry at the MIT Media Lab ${ }^{1}$, where a pocket projector, a mirror, a camera and a mobile computer are

${ }^{1}$ http: / / www . pranavmistry.com/projects/sixthsense/ 
involved. Other researchers are working on capturing and keeping track of human action at real time using computer graphics and hardware capabilities, built-in a computer. It transfers human motion parameters to a physical model to reproduce the movements of a target in a virtual scene ${ }^{2}$.

Some of the most prominent problems with the current research and prototypes of the motion recognition and recording systems are their high costs due to the usage of expensive sensors and some other high-end equipment, heavy weight computations due to complex recognition algorithms ${ }^{3}$. Some occasions only need to monitor basic actions of the monitored subject, and some cases may only allow to use simple camera to conduct monitoring. Therefore, there need solutions that can make use of cheap and widely available sensors to provide feasible solutions that can be widely adopted for normal every usage.

Considering all these issues, we tried to combine the convenience of SixthSence with some simple algorithms based on computer vision, and to use 3D model action library to present the recorded human actions. Then a comprehensive 3D model based action recording system using computer visions that can work almost anywhere is implemented, where the action recognition and recording is realized using an ordinary network camera and a simple algorithm to reduce costs and resource consumptions. The accuracy of action recognition is enhanced by 3D model actions in a system action library to express new actions.

The rest of the paper is structured as follows: Section 2 presents the design of the action recognition and recording system. Then Section 3, 4, 5 and 6 present how the system works include static background obtaining and updating, characters capturing, action tracking, 3D modeling and OGRE control judgment. Section 7 presents our evaluations in terms of performance and accuracy of recognitions. We compare our work with the related work in Section 8. Conclusions and future work end the paper.

\section{Design of an Action Recognition and Recording System}

Our action recognition and recording system mainly include the processes of background capturing, characters capturing, action tracking, 3D modeling and OGRE controlling, as shown in Figure 1.

At first in order to obtain a human body that appeared in a scene later, a network camera is used to capture static scene as the basis for moving objects detection. The system background is updated continuously.

Then in the characters capturing step, when a human enters the scene, the system detects the changing area of the image sequences and picks up the motion target from the background image. Usually only the pixel area corresponding to the motion target is considered in the motion tracking process.

After human characters are captured, the system starts to track the extracted detection target using the Camshift algorithm [5][6]. In this step, at first the system considers color information of a tracking target, this color information is used as

\footnotetext{
2 http: / /www.pranavmistry.com/projects/sixthsense/

${ }^{3}$ http://en.wikipedia.org/wiki/Motion_capture
} 
features of the tracking target, which is projected to the next frame of images to obtain the target. Then this frame of image is considered as a new source image for its immediate next frame. This process is repeated to achieve continuous tracking.

Finally the information captured from these early steps is expressed using a 3D model. We setup up bones binding where the skeletons are adjusted to the same size of the 3D model. After that, a 3D actions library is built to store different actions of characters. When a human motion is captured in a scene, the $3 \mathrm{D}$ model reacted with a corresponding action according to the captured color information. Then the system extracts animations from this 3D model entity and redraws this motion.

OpenCV (Open Source Computer Vision Library) is used for background and characters capturing. A background difference algorithm is used to analyze the moving target and extract different elements. For action tracking, the Camshift tracking algorithm is used to realize continuous tracking and recognition of moving objects and ensure the continuity of the action recorder [1]. 3DMAX is used to build skeletal animations. The skeletal animations are imported into the testing environment using OGRE APIs, which are controlled according to the captured features to realize the recording of body movement. We can see the detailed process as in Figure 1.

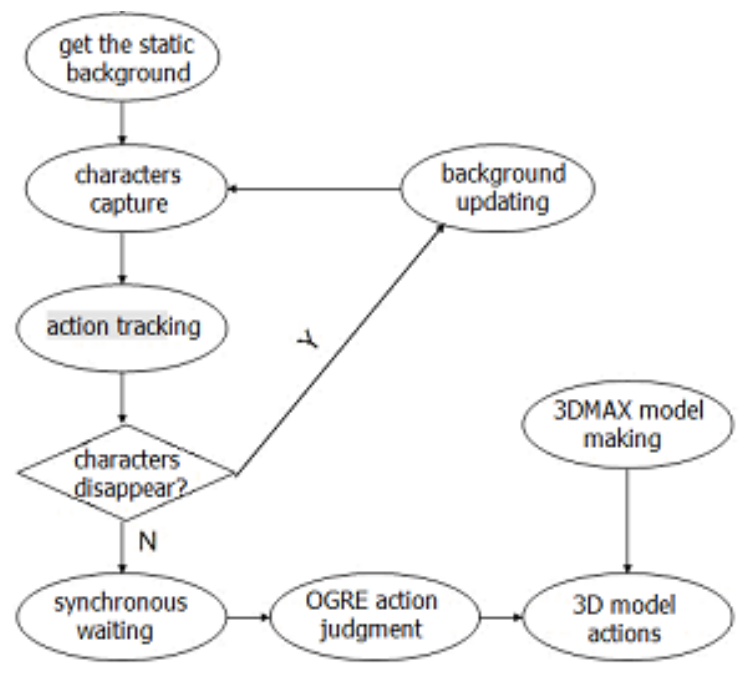

Fig. 1. Work flow of the action recognition and recording system

\section{Obtaining Static Background and Background Updating}

There are several ways to update background, including multi-frames averaging method, select update method, random update method and so on [2]. The multiframes averaging method is simple and accurate, but it costs too many resources. The select update method has strong adaptability to light changes, but it is error prone when there is a big light change [2]. 
Henceforth, in our system, pixels changes are obtained by a series of frame differences calculated from the comparisons of the frames with the background image. In this way, we can avoid the complexities of multi-frames averaging method [3][4], and provide acceptable light adaptability at the same time. If the pixels changes exceed a certain threshold, the original background image should be fused with the current background image to generate an optimized background image. This approach can help to detect whether there are moving objects and whether the background needs to be updated.

\section{Characters Capture}

There are many target detection methods, including the algorithm of frame difference, optical flow method, background difference method and so on [3]. We choose background difference method to analyze potential targets in a static background. The background difference method [3][4] requires certain differences between the grayscale values of target pixels and the background pixels (the camera should be still), i.e. it compares the target image with the background image to segment the target out, as calculated from Formula (1) and Formula (2).

$$
\begin{gathered}
D(x, y)=|C(x, y)-B(x, y)| \\
B W(x, y)=\left\{\begin{array}{l}
1, \text { if }(D(x, y) \geq T) \\
0, \text { if }(D(x, y)<T)
\end{array}\right\}
\end{gathered}
$$

The $\mathrm{B}(\mathrm{x}, \mathrm{y})$ stands for the background grayscale image, $\mathrm{C}(\mathrm{x}, \mathrm{y})$ for the current frame image, $\mathrm{D}(\mathrm{x}, \mathrm{y})$ for the difference image and $\mathrm{BW}(\mathrm{x}, \mathrm{y})$ for the binary image. $\mathrm{T}$ is the threshold value according to background noise or other factors to make distinctions between changes caused by target and background noise changes.

We can get the binary image and mark its connected area using Formula (2). Then with this connected area, we can make decisions on whether the connected area is a noise zone, or a moving target, based on its pixels number. If this number exceeds a certain threshold, it is a moving target. Or else, the connected area is a noise zone.

In this paper, the system processes the captured images when people enter the static view and detects the human part in which the pixel difference is greater than a threshold set in advance. As shown in the Figure 2, the black part is the background

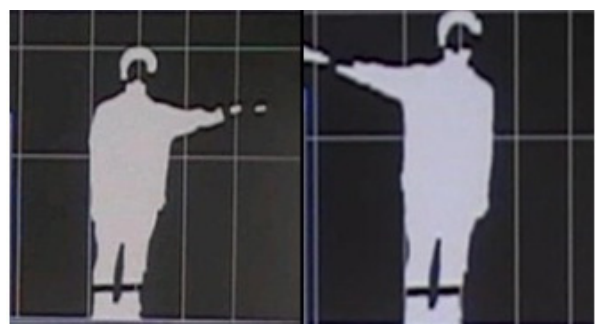

Fig. 2. Background Difference Processing Rendering 
after difference, the white part is the human body detected from the initial image, and the background difference method can detect the moving targets correctly. This background difference algorithm only uses brightness values as the detection basis, and if the brightness of the moving target is similar to the brightness of the background, it will be difficult to detect the moving target.

\section{$5 \quad$ Action Tracking}

The Camshift algorithm [5][6] has good reliability and can meet real time requirements, which can be used to find a target by color matching, and the color changes are small when the target is moving. Therefore Camshift is used in our project.

At first Camshift method transforms the RGB space to the HSV space and extracts $\mathrm{H}$ color component, then it extracts the tracking window and gets the selection zone. After that, the color histogram is used to transform the original image into color probability distribution images. Before processing, each pixel value in the target image describes the color information for that point. After processing, each pixel value means the possibility discretization measurement of the color information in this place. Then after searching from back projection of the target histogram, the system returns a tracking window of next frame. Finally, the system draws the position of the tracking results and loops through this execution.

\section{3D Model Actions and OGRE Action Judgment}

At first we take the Miku model into 3Dmax, where we set up bones binding and use bone cover technology to control the model. The skeleton is adjusted to the same size to match the Miku model, and all pixel points of the model are embedded into the bone cover, then the bind pose at each index refers to the bone with the same index, as Figure 3 shows.

Then the character model is hidden and only the initial state of the skeleton is left. With the normal playing speed set to the rate of 10 frames per second, the skeleton is adjusted to the optimum time point to make an alteration in order to build the model action. The animation is created when a series of points are operated in such a way, as shown in Figure 4. Then the model can be exported by OGRE.

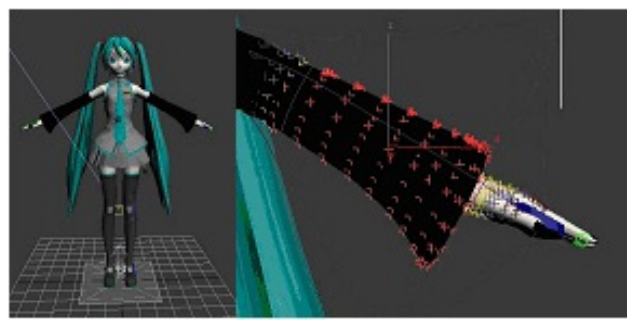

Fig. 3. Miku Model and Bone Binding 


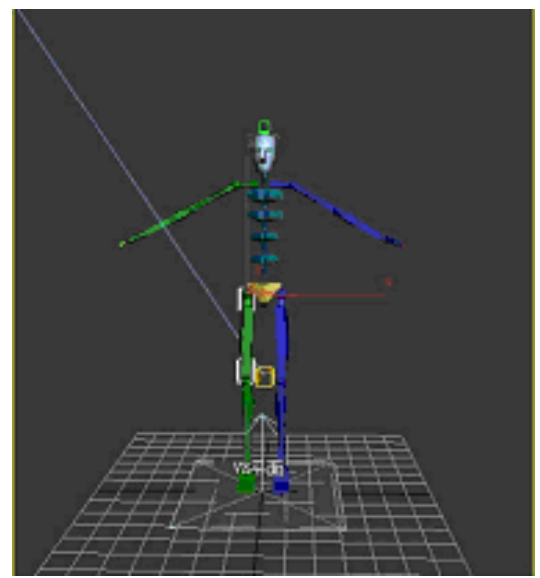

Fig. 4. Character Animation Process

The control part of OGRE is shown in Figure 5. OGRE extracts animations from the model entity after initializing the object model, then the model reacts to the data control with a corresponding action. After that the system uses the variables to record the current action and the animation is redrawn according to the corresponding value under the OGRE redraw mechanism.

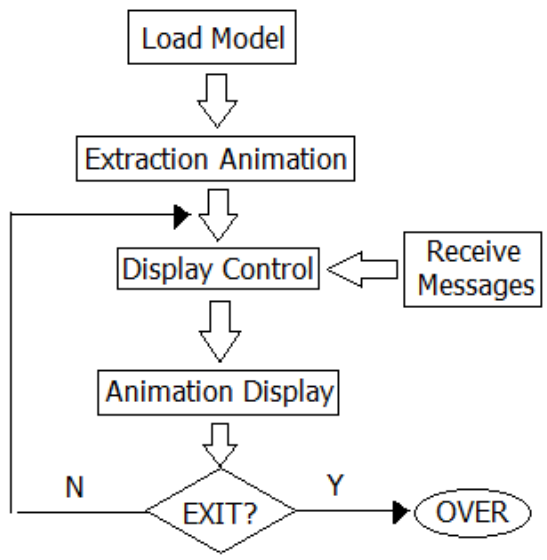

Fig. 5. OGRE Control Part Architecture

\section{Testing of the Action Recording System}

The system is tested on different platforms, an X86 computer (2GHz processor and $2 \mathrm{G}$ memory) and an ARM9 platform (S3c2410X platform, 203.3 $\mathrm{MHz}$ processor and $64 \mathrm{M}$ sdram). Ten tests were conducted, on average, the system response time is less than 1 second based on an X86 computer, and is less than 2 second based on an ARM9 platform. We show here the tests on an X86 computer. 
Table 1. Recognition of Different Actions and the corresponding performance

\begin{tabular}{|c|c|c|c|}
\hline Test Content & Test Results & Motion Effect & $\begin{array}{l}\text { System Delay } \\
\text { Average Time } \\
\text { (X86 / } 10 \text { times) }\end{array}$ \\
\hline $\begin{array}{l}\text { target } \\
\text { turns left }\end{array}$ & $\begin{array}{l}\text { model } \\
\text { turns left }\end{array}$ & & $\begin{array}{l}\text { target time: } 0 \mathrm{~s} \\
\text { track time: } 0.32 \mathrm{~s} \\
\text { model } \\
\text { time: } 0.43 \mathrm{~s} \\
\text { sum time: } 0.75 \mathrm{~s}\end{array}$ \\
\hline $\begin{array}{l}\text { target } \\
\text { turns right }\end{array}$ & $\begin{array}{l}\text { model } \\
\text { turns right }\end{array}$ & & $\begin{array}{l}\text { target time: } 0 \mathrm{~s} \\
\text { track time: } 0.34 \mathrm{~s} \\
\text { model } \\
\text { time: } 0.42 \mathrm{~s} \\
\text { sum time: } 0.76 \mathrm{~s}\end{array}$ \\
\hline $\begin{array}{l}\text { target } \\
\text { squatts } \\
\text { down }\end{array}$ & $\begin{array}{l}\text { model } \\
\text { squatts down }\end{array}$ & & $\begin{array}{l}\text { target time: } 0 \mathrm{~s} \\
\text { track time: } 0.29 \mathrm{~s} \\
\text { model } \\
\text { time: } 0.38 \mathrm{~s} \\
\text { sum time: } 0.67 \mathrm{~s}\end{array}$ \\
\hline $\begin{array}{l}\text { target lifts } \\
\text { and put } \\
\text { down the } \\
\text { left hand }\end{array}$ & $\begin{array}{l}\text { model lifts } \\
\text { and put } \\
\text { down the left } \\
\text { hand }\end{array}$ & & $\begin{array}{l}\text { target time: } 0 \mathrm{~s} \\
\text { track time: } 0.24 \mathrm{~s} \\
\text { model } \\
\text { time: } 0.34 \mathrm{~s} \\
\text { sum time: } 0.58 \mathrm{~s}\end{array}$ \\
\hline $\begin{array}{l}\text { target lifts } \\
\text { and puts } \\
\text { down the } \\
\text { right hand }\end{array}$ & $\begin{array}{l}\text { model lifts } \\
\text { and puts } \\
\text { down the } \\
\text { right hand }\end{array}$ & & $\begin{array}{l}\text { target time: } 0 \mathrm{~s} \\
\text { track time: } 0.25 \mathrm{~s} \\
\text { model } \\
\text { time: } 0.35 \mathrm{~s} \\
\text { sum time: } 0.60 \mathrm{~s}\end{array}$ \\
\hline
\end{tabular}

In our tests, the computer captures and tracks the character movement through a network camera, and manipulates the 3D girl [7] to perform corresponding actions as shown in table 1 . We can see that all actions can be correctly recognized, and the performance of the recognition process for all the actions is around $0.67 \mathrm{~s}$ on average.

The system preprocesses the captured image in order to improve performance and recognition accuracy. The preprocessing includes color space transformation, noise reduction and background updates. As shown in table 2, the system requires that the 
background remains relatively fixed, and also requires certain intensity of illumination, and no motion impurity in the background. The effect of background disturbance, illumination changes and shadows are the problems that must be considered in background difference method, and will be explored in our future work.

Table 2. Testing results

\begin{tabular}{|c|c|c|c|}
\hline Number & Test Environment & Conclusion & Analysis \\
\hline 1 & $\begin{array}{l}\text { Indoor, light on } \\
\text { and no moving } \\
\text { objects }\end{array}$ & good effects & $\begin{array}{l}\text { Under the fixed background and } \\
\text { no impurities, light }>2001 x, \\
\text { denoising effect is good. }\end{array}$ \\
\hline 2 & $\begin{array}{l}\text { Indoor, light on } \\
\text { and more than } 10 \\
\text { moving objects }\end{array}$ & $\begin{array}{l}\text { modest } \\
\text { effects }\end{array}$ & $\begin{array}{l}\text { Under the fixed background and } \\
\text { more than } 10 \text { impurities, light }> \\
\text { 2001x, denoising effect is } \\
\text { modest. }\end{array}$ \\
\hline 3 & $\begin{array}{l}\text { Indoor, light off } \\
\text { and no moving } \\
\text { objects }\end{array}$ & $\begin{array}{l}\text { modest } \\
\text { effects }\end{array}$ & $\begin{array}{l}\text { Under the fixed background and } \\
\text { no impurities, light }<501 x \text {, } \\
\text { denoising effect is modest. }\end{array}$ \\
\hline 4 & $\begin{array}{l}\text { Indoor, light off } \\
\text { and more than } 10 \\
\text { moving objects }\end{array}$ & $\begin{array}{l}\text { modest } \\
\text { effects }\end{array}$ & $\begin{array}{l}\text { Under the fixed background and } \\
\text { more than } 10 \text { impurities, light }< \\
501 x \text {, denoising effect is } \\
\text { modest. }\end{array}$ \\
\hline 5 & $\begin{array}{l}\text { Outdoor, light well } \\
\text { and more than } 10 \\
\text { moving objects }\end{array}$ & bad effects & $\begin{array}{c}\text { Under the unfixed background } \\
\text { and more than } 10 \text { impurities, } \\
\text { light }>2001 x \text {, denoising effect } \\
\text { is bad. }\end{array}$ \\
\hline
\end{tabular}

\section{$8 \quad$ Related Work}

Because human motion capture technology can connect physical world to the electronic world more expediently and effectively, it can be applied to healthcare systems, human-computer interaction, virtual reality systems and many other applications. Due to the availability of cheap sensors such as network camera, there arise needs for useful and simple solutions that have good performance.

Human motion capture technology can be evaluated according to the following criteria: location accuracy, real-time, convenience, cost, anti-jamming and capture range size. In [8][9], although they provided very accurate methods, the capture systems based on sensors are often limited by specialized sensors. For example, in [8] CCD sensors need complex external control circuits to support them, and their speed were not very ideal. In [9] wireless sensors needed a lot of calculation to determine their positions and relationships, and only the skeleton could be extracted in its system. In this paper, we proposed a solution with low requirements and costs for hardware and software, with acceptable performance. 
In [11], an improved real-time background update algorithm was proposed. They avoided the influence caused by the variety of background according to the $3 \sigma$ rule of normal distribution, and proposed a shadow removing algorithm based on the vertical projection. Shadow disappearance is not the best display method, so we used the 3D model and shows the performance is better, where OpenCV effect and 3D model used together for visual display.

Similarly, background difference algorithm was used in [10]. They introduced omnidirectional camera to tracking multiple targets in indoor environments, and built the background model by observing the scene without people first. When the detecting process started, omnidirectional images were changed into the cylindrical panoramic images, and an adaptive background subtraction method was utilized to segment the moving regions and locate the positions of human bodies. The heavy computation would consume more resources. We used very common network camera in our system.

A modified mixture Gaussian model was used in [12]. In their method, foreground objects were segmented based on an improved binary connected component analysis and Kalman filtering was used for object tracking. In [13], a zero Mean normalized cross-correlation method was proposed to achieve the detection of moving targets, and the cumulative histogram method was used to obtain a satisfactory threshold to achieve a precise movement of the extraction of the target under complex background enviroment. Our system requires a certain intensity of illumination and no motion impurity in the background, so the methods in [12] and [13] could be introduced to our system to improve adaptability to lighting changes, shadows and occlusions.

\section{Conclusions and Future Work}

In the motion recording system proposed in our paper, we used computer vision (OpenCV library) for human body motion capture. With background difference algorithm and Camshift algorithm, the system captures the human motion video by camera and gets the data results by the detection and tracking of the video resources, then the 3D model is controlled by the captured feature to record the action of human.

The system obtains visual images directly and compares the images with 3D model at real time. The system requires the static scene to build the background model, then the background difference method and the Camshift method are used for detecting and tracking the moving object, so it requires that the environment has enough light (>2001x) and fewer objects. In our system, it is easy to track the bright objects rather than white objects due to low $\mathrm{H}$ components in white ones. The tests show that the recording system has good performance and can recognize different actions correctly. The system works well and no lag and jumping frame had detected.

In the future, more cameras will be used to improve its accuracy and anti-jamming capability. With more cameras, multi-angle images information would be obtained to locate the target actions more precisely and to discard impurity elements. In addition, we could improve the accuracy of the $3 \mathrm{D}$ model to solve this problem. Then when a new action appeared, the related 3D model action should be edited to join the system action library to express new human motions. 
Acknowledgments. Weishan Zhang has been supported by "the Fundamental Research Funds for the Central Universities" and also the start up funds for "Academic Top-Notch in China University of Petroleum" professors.

\section{References}

1. Mao, J., Zou, X., Zhu, J.: Improved Plane Template Two-step Camera Calibration. China Journal of Image and Graphics 9(7), 846-852 (2004)

2. Zhang, C., Su, Y.: Digital Image Compression, pp. 20-23. Tsinghua University Press (2006)

3. Park, J.S.: Interactive 3D reconstruction from multiple images: a primitive-based approach. Pattern Recognition Letters 26(16), 2558-2571 (2005)

4. Intel Corporation. Open Source Computer Vision Library Reference Manual (December 2001)

5. de la Torre Frade, F., Marroquín, E.M., Pérez, M.E.S., Moreno, J.A.M.: Moving object detection and tracking system: a real-time implementation (1997)

6. Nascimento, J.C., Marques, J.S.: Performance evaluation of object detection algorithms for video surveillance. IEEE Trans. Multimedia, 761-774 (2006)

7. Wu, F., Li, H., Hu, Z.: A New Camera Self-calibration Method Based on Active Vision System. Chinese Journal of Computers 23(11), 1130-1139 (2000)

8. Feng, L., Wang, R.: Application of CCD Sensors in the Image Detection System of Human Motion. Instrument Technique and Sensor 5, 33-35 (2000)

9. Wang, T., Feng, W., Luo, D.: Application of Wireless Sensor Network for Detection of Human Body Movements. Instrumentation and Measurment 28(8), 83-86 (2009)

10. Pi, W., Liu, H., Cha, H.: Motion Detection for Human Bodies Basing Adaptive Background Subtraction by Using an Omnidirectional Camera. Journal of Peking University 40(3), 458-464 (2004)

11. Cao, D., Zou, W., Wu, Y.: Motion human detection based on difference background image. Opto-Electronic Engineering 34(6), 107-111 (2007)

12. Liu, Y., Ai, H., Xu, G.: Moving object detection and tracking based on background subtraction. Information and Control 31(8), 315-319 (2002)

13. Wei, J., Liu, Z., Zhang, H.: Moving objects detection algorithm under complex background environment. Microcomputer \& Its Applications 2, 49-51 (2010) 